\title{
Filter Design for Continuous-Time Linear Systems Subject to Sensor Saturation
}

\author{
Márcio J. Lacerda \\ Department of Electrical Engineering, Federal University of São João del Rei (UFSJ), 36307-352 São João del Rei, MG, Brazil \\ Correspondence should be addressed to Márcio J. Lacerda; lacerda@ufsj.edu.br
}

Received 24 January 2017; Revised 19 May 2017; Accepted 28 May 2017; Published 28 June 2017

Academic Editor: Raquel Caballero-Águila

Copyright ( 2017 Márcio J. Lacerda. This is an open access article distributed under the Creative Commons Attribution License, which permits unrestricted use, distribution, and reproduction in any medium, provided the original work is properly cited.

\begin{abstract}
This paper presents new sufficient conditions to cope with the filtering problem for continuous-time linear systems subject to sensor saturation. A generalized sector condition has been used to handle the saturation in the measured output. The $\mathscr{H}_{\infty}$ performance was considered and a quadratic Lyapunov function was employed in order to derive the design conditions. The conditions are presented in terms of matrix inequalities that become linear when a scalar parameter is fixed. The efficiency of the proposed conditions and their capability to deal with different levels of saturation are illustrated by numerical examples.
\end{abstract}

\section{Introduction}

The study of nonlinear phenomena occurring in control systems has attracted a lot of attention in the last years, mainly because of the effects these types of phenomena may cause [1]. Among others, one may cite quantization, time-delay, polynomial systems, hysteresis, and saturation [2-6]. The presence of those effects can degrade the performance and even bring instability to the control systems. Therefore, one must be cautious when analyzing systems that are subjected to nonlinear effects. The saturation phenomenon is related to the physical limitations presented in actuators, sensors, and other systems components. When the presence of saturation is not taken into account in the design process, it can lead to very conservative performances $[4,7,8]$.

In the control literature, one of the most studied topics is the filter design problem [9]. In the filtering problem, we are interested in obtaining a good approximation of a desired signal in the presence of noise. The filter to be designed makes use of the output measurements of the system to provide an estimation of the desired signal. The desired signal can be a combination of the states or the states themselves. The problems have been studied under different conditions such as linear systems [10], uncertain systems [11, 12], timedelay systems $[13,14]$, polynomial systems $[2,15]$, and $2 \mathrm{D}$ systems [16]. Although the filter design depends on the output measurements of the system, which are obtained by using sensors that can present saturation, this scenario has not been fully explored in the literature.

In [17], the $\mathscr{H}_{\infty}$ filtering problem has been studied for continuous-time systems subject to sensor nonlinearities, including sensor saturation. The main difference between the proposed technique and the method presented in [17] resides in the sector condition that has been employed. This paper addresses the problem of robust linear filter design for continuous-time linear systems subject to sensor saturation. The $\mathscr{H}_{\infty}$ performance will be used as performance criteria and a generalized sector condition will be employed to handle the saturation. A quadratic Lyapunov function is considered and the filter design is accomplished by partitioning the Lyapunov matrix and its inverse. New sufficient conditions in the form of matrix inequalities are presented for both nominal case and uncertain case. Numerical experiments from the literature illustrate the potential of the proposed conditions and indicate that the saturation in the measured output does affect the $\mathscr{H}_{\infty}$ performance of the robust linear filter.

This paper is organized as follows. Section 2 introduces the problem under consideration and the main goal of this paper. Section 3 presents some preliminary results and the main results are given in Section 4. Section 5 is devoted to present the numerical experiments and Section 6 concludes the paper. 
Notation 1. For two symmetric matrices of the same dimensions $X$ and $Y, X>Y$ means that $X-Y$ is positive definite. $\mathbb{R}^{+}$ is the set of positive real numbers. For matrices and vectors, $\left({ }^{\prime}\right)$ indicates the transpose. Identity matrices are denoted by $I$ and null matrices are denoted by 0 . The symbol $\star$ indicates a symmetric block in matrices.

\section{Problem Statement}

Consider the following continuous-time system:

$$
\begin{aligned}
& \dot{x}(t)=A x(t)+B w(t), \\
& z(t)=C_{z} x(t)+D_{z} w(t), \\
& y(t)=\operatorname{sat}_{y_{0}}\left(C_{y} x(t)\right),
\end{aligned}
$$

where $x \in \mathbb{R}^{n}$ is the state vector, $w \in \mathbb{R}^{n_{w}}$ is the noise input, $z \in \mathbb{R}^{n_{z}}$ is the signal to be estimated, $y \in \mathbb{R}^{n_{y}}$ is the measured output, and $t \in \mathbb{R}^{+}$is the time domain. The constant matrices that describe the system have the following dimensions: $A \in \mathbb{R}^{n \times n}, B \in \mathbb{R}^{n \times n_{w}}, C_{z} \in \mathbb{R}^{n_{z} \times n}, D_{z} \in \mathbb{R}^{n_{z} \times n_{w}}$, and $C_{y} \in \mathbb{R}^{n_{y} \times n}$. The matrix $A$ is supposed to be Hurwitz stable. Due to physical limitations, the sensor output $y$ is supposed to be bounded in amplitude; that is,

$$
-y_{0(i)} \leq y_{(i)} \leq y_{0(i)}, \quad y_{0(i)}>0, \quad i=1, \ldots, n_{y}
$$

Furthermore, the signal $w$ is supposed to be energy bounded; that is, $w \in \mathscr{L}_{2}$. Without loss of generality, we assume that the signal $w$ is $\mathscr{L}_{2}$-normalized; that is, it satisfies

$$
\|w\|_{2}^{2}=\int_{0}^{\infty} w(\tau)^{\prime} w(\tau) d \tau \leq 1 .
$$

The problem to be addressed in this paper is finding a full order stable robust continuous-time linear filter given by

$$
\begin{aligned}
& \dot{x}_{f}(t)=A_{f} x_{f}(t)+B_{f} y(t), \\
& z_{f}(t)=C_{f} x_{f}(t)+D_{f} y(t),
\end{aligned}
$$

where $x_{f} \in \mathbb{R}^{n_{f}}, n_{f}=n$, is the estimated state and $z_{f} \in \mathbb{R}^{n_{z}}$ is the estimated output, and the filtering matrices are $A_{f} \in$ $\mathbb{R}^{n_{f} \times n_{f}}, B_{f} \in \mathbb{R}^{n_{f} \times n_{y}}, C_{f} \in \mathbb{R}^{n_{z} \times n_{f}}$, and $D_{f} \in \mathbb{R}^{n_{z} \times n_{y}}$. As a consequence of the bounds (2), the output $y$ that will be used by the filter is a saturated one

$$
y(t)=\operatorname{sat}_{y_{0}}\left(C_{y} x(t)\right)
$$

and each component of $\operatorname{sat}\left(C_{y} x(t)\right)$ is defined, $\forall i=1, \ldots, n_{y}$, by

$$
\begin{aligned}
\operatorname{sat} & \left(C_{y} x(t)\right) \\
\triangleq & \operatorname{sign}\left(C_{y(i)} x(t)\right) \min \left(\left|C_{y(i)} x(t)\right|, y_{0(i)}\right) .
\end{aligned}
$$

Defining the decentralized dead-zone nonlinearity $\phi \in \mathbb{R}^{n_{y}}$ as

$$
\phi(x)=\operatorname{sat}_{y_{0}}\left(C_{y} x(t)\right)-C_{y} x(t),
$$

system (1) reads

$$
\begin{aligned}
& \dot{x}(t)=A x(t)+B w(t), \\
& z(t)=C_{z} x(t)+D_{z} w(t), \\
& y(t)=\phi+C_{y} x(t) .
\end{aligned}
$$

Connecting filter (4) with system (8) and defining the augmented state vector $\tilde{x}(t)^{\prime}=\left[x(t)^{\prime} x_{f}(t)^{\prime}\right]$ and the output error $e(t)=z(t)-z_{f}(t)$, one has

$$
\begin{aligned}
& {\left[\begin{array}{c}
\dot{x}(t) \\
\dot{x}_{f}(t)
\end{array}\right]=\left[\begin{array}{cc}
A & 0 \\
B_{f} C_{y} & A_{f}
\end{array}\right]\left[\begin{array}{c}
x(t) \\
x_{f}(t)
\end{array}\right]+\left[\begin{array}{l}
B \\
0
\end{array}\right] w(t)+\left[\begin{array}{c}
0 \\
B_{f}
\end{array}\right] \phi,} \\
& e(t)=\left[C_{z}-D_{f} C_{y}-C_{f}\right]\left[\begin{array}{c}
x(t) \\
x_{f}(t)
\end{array}\right]+\left[D_{z}\right] w(t)+\left[-D_{f}\right] \phi
\end{aligned}
$$

that can be written in a compact form with

$$
\begin{aligned}
& \dot{\tilde{x}}(t)=\widetilde{A} \widetilde{x}(t)+\widetilde{B} w(t)+\widetilde{B}_{s} \phi, \\
& e(t)=\widetilde{C} \widetilde{x}(t)+\widetilde{D} w(t)+\widetilde{D}_{s} \phi,
\end{aligned}
$$

where

$$
\begin{aligned}
\widetilde{A} & =\left[\begin{array}{cc}
A & 0 \\
B_{f} C_{y} & A_{f}
\end{array}\right] \in \mathbb{R}^{2 n \times 2 n}, \\
\widetilde{B} & =\left[\begin{array}{l}
B \\
0
\end{array}\right] \in \mathbb{R}^{2 n \times n_{w}}, \\
\widetilde{B}_{s} & =\left[\begin{array}{c}
0 \\
B_{f}
\end{array}\right] \in \mathbb{R}^{2 n \times n_{y}}, \\
\widetilde{C} & =\left[C_{z}-D_{f} C_{y}-C_{f}\right] \in \mathbb{R}^{n_{z} \times 2 n}, \\
\widetilde{D} & =\left[D_{z}\right] \in \mathbb{R}^{n_{z} \times n_{w}}, \\
\widetilde{D} & =\left[-D_{f}\right] \in \mathbb{R}^{n_{z} \times n_{y}} .
\end{aligned}
$$

The $\mathscr{H}_{\infty}$ performance will be used as the performance criteria, assuring that the augmented system (10) is asymptotically stable and the energy gain from the disturbance input $w(t)$ to the error $e(t)=z(t)-z_{f}(t)$ is minimized.

Definition 2 (see [18]). If there exists a Lyapunov function $V(\widetilde{x}(t))>0$, then a bound $\gamma^{2}$ to the $\mathscr{H}_{\infty}$ performance of the augmented system (10), from the noise input $w(t)$ to the error output $e(t)$, can be obtained by

$$
\dot{V}(\tilde{x}(t))+\frac{1}{\gamma^{2}} e(t)^{\prime} e(t)-w(t)^{\prime} w(t)<0 .
$$

\section{Preliminaries}

The preliminary results presented here follow the lines in [19, 20]. 
Proposition 3. If there exist a matrix $Q=Q^{\prime}>0 \in \mathbb{R}^{2 n \times 2 n}$, a diagonal matrix $S_{1}>0 \in \mathbb{R}^{n_{y} \times n_{y}}$, and $F \in \mathbb{R}^{n_{y} \times 2 n}$ such that the inequalities

$$
\begin{aligned}
& {\left[\begin{array}{cccc}
Q \widetilde{A}^{\prime}+\widetilde{A} Q & \widetilde{B} & \widetilde{B}_{s} S_{1}-Q \widetilde{C}_{s}^{\prime}-F^{\prime} & Q \widetilde{C}^{\prime} \\
\star & -I_{n_{w}} & 0 & \widetilde{D}^{\prime} \\
\star & \star & -2 S_{1} & S_{1} \widetilde{D}_{s}^{\prime} \\
\star & \star & \star & -\gamma^{2} I_{n_{z}}
\end{array}\right]<0,} \\
& {\left[\begin{array}{cc}
Q & F^{\prime}(j) \\
\star & y_{0(j)}^{2}
\end{array}\right] \geq 0 \text {, }} \\
& j=1, \ldots, n_{y}
\end{aligned}
$$

are satisfied, then the $\mathscr{H}_{\infty}$ performance is limited by $\gamma$ for every initial condition belonging to $\mathscr{E}\left(Q^{-1}\right)=\{\widetilde{x}(t) \in$ $\left.\mathbb{R}^{2 n} ; \widetilde{x}(t)^{\prime} Q^{-1} \widetilde{x}(t) \leq 1\right\}$.

Proof. Consider the quadratic Lyapunov function $V(\widetilde{x}(t))=$ $\widetilde{x}(t)^{\prime} Q^{-1} \widetilde{x}(t)$, with $Q=Q^{\prime}>0$. The $\mathscr{H}_{\infty}$ performance bound from $w(t)$ to $e(t)$ for system (10) can be obtained by

$$
\dot{V}(\widetilde{x}(t))+\frac{1}{\gamma^{2}} e(t)^{\prime} e(t)-w(t)^{\prime} w(t)<0 .
$$

By using equations from system (10), one can write

$$
\begin{gathered}
{\left[\begin{array}{c}
\widetilde{x}(t) \\
w(t) \\
\phi
\end{array}\right]^{\prime}\left(\left[\begin{array}{ccc}
\widetilde{A}^{\prime} Q^{-1}+Q^{-1} \widetilde{A} & Q^{-1} \widetilde{B} & Q^{-1} \widetilde{B}_{s} \\
\widetilde{B}^{\prime} Q^{-1} & -I_{n_{w}} & 0 \\
\widetilde{B}_{s}^{\prime} Q^{-1} & 0 & 0
\end{array}\right]\right.} \\
\left.+\left[\begin{array}{c}
\widetilde{C}^{\prime} \\
\widetilde{D}^{\prime} \\
\widetilde{D}_{s}^{\prime}
\end{array}\right] \frac{1}{\gamma^{2}} I_{n_{z}}\left[\begin{array}{ccc}
\widetilde{C} & \widetilde{D} & \widetilde{D}_{s}
\end{array}\right]\right)\left[\begin{array}{c}
\widetilde{x}(t) \\
w(t) \\
\phi
\end{array}\right]<0 .
\end{gathered}
$$

Given that $\widetilde{x}(t) \in S\left(y_{0}\right)$ with $S\left(y_{0}\right)=\left\{\widetilde{x}(t) \in \mathbb{R}^{n} ;-y_{0} \leq\right.$ $\left.F Q^{-1} \tilde{x}(t) \leq y_{0}\right\}$, one can use Lemma 1 from [6], to verify that

$$
-2 \phi^{\prime} S_{1}^{-1}\left(\phi+C_{y} x(t)-F Q^{-1} \tilde{x}(t)\right) \geq 0,
$$

where $S_{1}$ is a positive diagonal matrix. It is possible to rewrite (17) as

$$
-2 \phi^{\prime} S_{1}^{-1}\left(\phi+\bar{C}_{s} \tilde{x}(t)-F Q^{-1} \tilde{x}(t)\right) \geq 0,
$$

where $\bar{C}_{s}=\left[\begin{array}{ll}C_{y} & 0_{n_{y} \times n}\end{array}\right]$. Applying a Schur complement on inequality (14), one can write

$$
Q-F^{\prime} \frac{1}{y_{0}^{2}} F \geq 0 .
$$

Pre- and postmultiplying the last inequality by $Q^{-1}$, one has

$$
Q^{-1}-Q^{-1} F^{\prime} \frac{1}{y_{0}^{2}} F Q^{-1} \geq 0
$$

or equivalently

$$
\widetilde{x}(t)^{\prime} Q^{-1} \tilde{x}(t) \geq \widetilde{x}(t)^{\prime} Q^{-1} F^{\prime} \frac{1}{y_{0}^{2}} F Q^{-1} \widetilde{x}(t) .
$$

In this way, one concludes that the ellipsoid $\mathscr{E}\left(Q^{-1}\right)=\{\tilde{x} \in$ $\left.\mathbb{R}^{2 n} ; \tilde{x}^{\prime} Q^{-1} \tilde{x} \leq 1\right\}$ is contained in $S\left(y_{0}\right)$. Moreover, if $(18)$ is positive definite, one can write

$$
\begin{aligned}
\dot{V}(\widetilde{x}(t)) & +\frac{1}{\gamma^{2}} e(t)^{\prime} e(t)-w(t)^{\prime} w(t) \\
\leq & \dot{V}(\widetilde{x}(t))+\frac{1}{\gamma^{2}} e(t)^{\prime} e(t)-w(t)^{\prime} w(t) \\
& -2 \phi^{\prime} S_{1}^{-1}\left(\phi+\bar{C}_{s} \tilde{x}(t)-F Q^{-1} \tilde{x}(t)\right) .
\end{aligned}
$$

To ensure that (15) holds, it suffices to verify that the right side of inequality (22) is negative definite. This fact can be guaranteed by the following inequality:

$$
\begin{aligned}
& {\left[\begin{array}{c}
Q^{-1} \tilde{x}(t) \\
w(t) \\
S_{1}^{-1} \phi
\end{array}\right]^{\prime}\left(\left[\begin{array}{ccc}
R_{1} & \widetilde{B} & R_{2} \\
\widetilde{B}^{\prime} & -I_{n_{w}} & 0 \\
R_{2}^{\prime} & 0 & -2 S_{1}
\end{array}\right]\right.} \\
& \left.+\left[\begin{array}{c}
Q \widetilde{C}^{\prime} \\
\widetilde{D}^{\prime} \\
S_{1} \widetilde{D}_{s}^{\prime}
\end{array}\right] \frac{1}{\gamma^{2}} I_{n_{z}}\left[\begin{array}{lll}
\widetilde{C} Q & \widetilde{D} & \widetilde{D}_{s} S_{1}
\end{array}\right]\right)\left[\begin{array}{c}
Q^{-1} \widetilde{x}(t) \\
w(t) \\
S_{1}^{-1} \phi
\end{array}\right] \\
& <0 \text {, }
\end{aligned}
$$

where

$$
\begin{aligned}
& R_{1}=Q \widetilde{A}^{\prime}+\widetilde{A} Q, \\
& R_{2}=\widetilde{B}_{s} S_{1}-Q \widetilde{C}_{s}^{\prime}-F^{\prime} .
\end{aligned}
$$

By applying Schur complement in (23), one has condition (13):

$$
\left[\begin{array}{cccc}
R_{1} & \widetilde{B} & R_{2} & Q \widetilde{C}^{\prime} \\
\star & -I_{n_{w}} & 0 & \widetilde{D}^{\prime} \\
\star & \star & -2 S_{1} & S_{1} \widetilde{D}_{s}^{\prime} \\
\star & \star & \star & -\gamma^{2} I_{n_{z}}
\end{array}\right]<0 .
$$

\section{Main Results}

The following theorem presents a sufficient condition for the filter design problem based on Proposition 3.

Theorem 4. If there exist positive definite symmetric matrices $Z \in \mathbb{R}^{n \times n}$ and $X \in \mathbb{R}^{n \times n}$, a positive definite diagonal matrix $S_{1} \in \mathbb{R}^{n_{y} \times n_{y}}$, matrices $G \in \mathbb{R}^{n \times n}, H \in \mathbb{R}^{n_{z} \times n}, L \in \mathbb{R}^{n \times n_{y}}$, $W_{1} \in \mathbb{R}^{n \times n_{y}}, W_{2} \in \mathbb{R}^{n \times n_{y}}$, and $D_{f} \in \mathbb{R}^{n_{z} \times n_{y}}$, and a scalar $\gamma$ such that the following inequalities are satisfied, 


$$
\left[\begin{array}{ccccc}
A^{\prime} Z+Z A & A^{\prime} X+Z A+C_{y}^{\prime} L^{\prime}+G^{\prime} & Z B & -C_{y}^{\prime}-W_{1} & C_{z}^{\prime}-C_{y}^{\prime} D_{f}^{\prime}-H^{\prime} \\
\star & A^{\prime} X+X A+C_{y}^{\prime} L^{\prime}+L C_{y} & X B & L S_{1}-C_{y}^{\prime}-W_{2} & C_{z}^{\prime}-C_{y}^{\prime} D_{f}^{\prime} \\
\star & \star & -I_{n_{w}} & 0 & D_{z}^{\prime} \\
\star & \star & \star & -2 S_{1} & -S_{1} D_{f}^{\prime} \\
\star & \star & \star & \star & -\gamma^{2} I_{n_{z}}
\end{array}\right]<0,
$$

then

$$
\begin{gathered}
A_{f}=\left(U^{\prime}\right)^{-1} G(V Z)^{-1}, \\
B_{f}=\left(U^{\prime}\right)^{-1} L, \\
C_{f}=H(V Z)^{-1},
\end{gathered}
$$

are the matrices of the robust filter that guarantee an $\mathscr{H}_{\infty}$ performance bounded by $\gamma^{2}$. The matrices $U$ and $V$ are obtained from the relation $X Y+U^{\prime} V=I$.

Proof. Define

$$
\begin{aligned}
Q & =\left[\begin{array}{cc}
Y & V^{\prime} \\
V & \widetilde{Y}
\end{array}\right], \\
Q^{-1} & =\left[\begin{array}{cc}
X & U^{\prime} \\
U & \widetilde{X}
\end{array}\right], \\
Q^{-1} Q & =I,
\end{aligned}
$$

where $Y, \widetilde{Y}, X$, and $\widetilde{X}$ are positive definite symmetric matrices.

$$
\begin{gathered}
X Y+U^{\prime} V=I, \\
X V^{\prime}+U^{\prime} \widetilde{Y}=0, \\
U V^{\prime}+\widetilde{X} \widetilde{Y}=I, \\
U Y+\widetilde{X} V=0 .
\end{gathered}
$$

Define the matrices

$$
\begin{aligned}
S & =\left[\begin{array}{cc}
Y & I \\
V & 0
\end{array}\right], \\
S^{-1} & =\left[\begin{array}{ll}
0 & V^{-1} \\
I & -Y V^{-1}
\end{array}\right], \\
R & =\left[\begin{array}{ll}
Y & 0 \\
0 & I
\end{array}\right], \\
R^{-1} & =\left[\begin{array}{ll}
Y^{-1} & 0 \\
0 & I
\end{array}\right] .
\end{aligned}
$$

By multiplying (13) on the left by $T_{1}=\operatorname{diag}\left(R^{-1} S^{\prime} Q^{-1}, I, I, I\right)$ and on the right by $T_{1}^{\prime}$, one has condition (26) where

$$
\begin{aligned}
Z & =Y^{-1}, \\
L & =U^{\prime} B_{f}, \\
G & =U^{\prime} A_{f} V Z, \\
H & =C_{f} V Z, \\
W_{1} & =Z F_{1}^{\prime}, \\
W_{2} & =X F_{1}^{\prime}+U^{\prime} F_{2}^{\prime} .
\end{aligned}
$$

By multiplying (14) on the left by $T_{2}=\operatorname{diag}\left(R^{-1} S^{\prime} Q^{-1}, I\right)$ and on the right by $T_{2}^{\prime}$, one has condition (27). For details about the filter reconstruction, the reader is referred to [21].

Remark 5. Note that Theorem 4 presents a bilinear matrix inequality condition, because the variable $S_{1}$ appears multiplying some other variables of the problem. However, since matrix $S_{1}$ is diagonal, this problem can be overcome by considering

$$
S_{1}=\operatorname{diag} \underbrace{(\lambda, \lambda, \ldots, \lambda)}_{p \text { times }}, \quad \lambda>0
$$

and performing a line search in $\lambda>0$. In this way, condition (26) becomes a linear matrix inequality for each fixed value 
of $\lambda$. Moreover, since matrix $S_{1}$ is precisely known, matrix $D_{f}$ will be recovered directly from condition (26) and no change of variables is necessary in this case.

The proposed approach can also be extended to deal with time-varying uncertainties and state-dependent polytopic uncertainties as introduced in [22]. In this case, the system is described as

$$
\begin{aligned}
& \dot{x}(t)=A(\sigma) x(t)+B(\sigma) w(t), \\
& z(t)=C_{z}(\sigma) x(t)+D_{z}(\sigma) w(t), \\
& y(t)=\phi+C_{y}(\sigma) x(t) .
\end{aligned}
$$

The matrices in (34) belong to a polytopic domain parameterized in terms of the states $x(t)$ and in terms of a time-varying parameter $\alpha$, being generically represented by

$$
Z(\sigma)=\sum_{i=1}^{N} \sigma_{i}\left(x^{i}, \alpha^{i}\right) Z_{i}, \quad \sigma \in \Lambda_{N}
$$

where $Z(\sigma)$ represents any matrix of the system in (34), $Z_{i}, i=1, \ldots, N$, are the vertices, $N$ is the number of vertices of the polytope, and $\Lambda_{N}$ is the unit simplex, given by

$$
\begin{aligned}
\Lambda_{N} & =\left\{\sigma \in \mathbb{R}^{N}: \sum_{i=1}^{N} \sigma_{i}\left(x^{i}, \alpha^{i}\right)=1, \sigma_{i}\left(x^{i}, \alpha^{i}\right) \geq 0, i\right. \\
& =1, \ldots, N\} .
\end{aligned}
$$

Connecting the filter as in (4) with system (34), one can use the same procedure as presented before to obtain sufficient conditions to design a robust filter for system (34). The next theorem presents the conditions.

Theorem 6. If there exist positive definite symmetric matrices $Z \in \mathbb{R}^{n \times n}$ and $X \in \mathbb{R}^{n \times n}$, a positive definite diagonal matrix $S_{1} \in \mathbb{R}^{n_{y} \times n_{y}}$, matrices $G \in \mathbb{R}^{n \times n}, H \in \mathbb{R}^{n_{z} \times n}, L \in \mathbb{R}^{n \times n_{y}}$, $W_{1} \in \mathbb{R}^{n \times n_{y}}, W_{2} \in \mathbb{R}^{n \times n_{y}}$, and $D_{f} \in \mathbb{R}^{n_{z} \times n_{y}}$, and a scalar $\gamma$ such that inequality (27) and the following inequalities are satisfied,

$$
\left[\begin{array}{ccccc}
A_{i}^{\prime} Z+Z A_{i} & A_{i}^{\prime} X+Z A_{i}+C_{y_{i}}^{\prime} L^{\prime}+G^{\prime} & Z B_{i} & -C_{y_{i}}^{\prime}-W_{1} & C_{z_{i}}^{\prime}-C_{y_{i}}^{\prime} D_{f}^{\prime}-H^{\prime} \\
\star & A_{i}^{\prime} X+X A_{i}+C_{y_{i}}^{\prime} L^{\prime}+L C_{y_{i}} & X B_{i}+L D_{y_{i}} & L S_{1}-C_{y_{i}}^{\prime}-W_{2} & C_{z_{i}}^{\prime}-C_{y_{i}}^{\prime} D_{f}^{\prime} \\
\star & \star & -I_{n_{w}} & 0 & D_{z_{i}}^{\prime} \\
\star & \star & \star & -2 S_{1} & -S_{1} D_{f}^{\prime} \\
\star & \star & \star & \star & -\gamma^{2} I_{n_{z}}
\end{array}\right]<0
$$

for $i=1, \ldots, N$, then

$$
\begin{gathered}
A_{f}=\left(U^{\prime}\right)^{-1} G(V Z)^{-1}, \\
B_{f}=\left(U^{\prime}\right)^{-1} L, \\
C_{f}=H(V Z)^{-1}, \\
D_{f}
\end{gathered}
$$

are the matrices of the robust filter that guarantee an $\mathscr{H}_{\infty}$ performance bounded by $\gamma^{2}$. The matrices $U$ and $V$ are obtained from the relation $X Y+U^{\prime} V=I$.

Proof. Multiplying condition (37) by $\sigma_{i}$ for $i=1, \ldots, N$ and summing up, one has a parameter dependent condition. Then, the proof follows the same steps as the ones in proof of Theorem 4.

The comments presented in Remark 5 are also valid for this case.

\section{Numerical Experiments}

The main goal of the experiments is to illustrate the potential of the proposed conditions and show the effect of the saturation level $y_{0}$ in the $\mathscr{H}_{\infty}$ performance of the robust filter designed by Theorems 4 and 6 . The routines were implemented in Matlab, version 7.1.0.246 (R14) SP 3 using the packages Yalmip [23] and SeDuMi [24].

Example 7. Consider the continuous-time system borrowed from [25] that describes the longitudinal dynamics of the F-8 aircraft, whose system matrices are

$$
A=\left[\begin{array}{cccc}
-2.4701 & 0.3430 & -1.2680 & -4.3661 \\
-2.5618 & 0.0539 & -17.2462 & -36.5734 \\
-2.0172 & 0.0698 & -2.5839 & -5.1006 \\
1 & 0 & 0 & 0
\end{array}\right]
$$$$
B=\left[\begin{array}{ll}
1 & 0 \\
0 & 1 \\
0 & 0 \\
0 & 0
\end{array}\right] \text {, }
$$

$$
\begin{aligned}
C_{y} & =\left[\begin{array}{cccc}
0 & 0 & 0 & 1 \\
0 & 0 & -1 & 1
\end{array}\right], \\
C_{z} & =\left[\begin{array}{llll}
0 & 0 & 0 & 1
\end{array}\right], \\
D_{z} & =0 .
\end{aligned}
$$




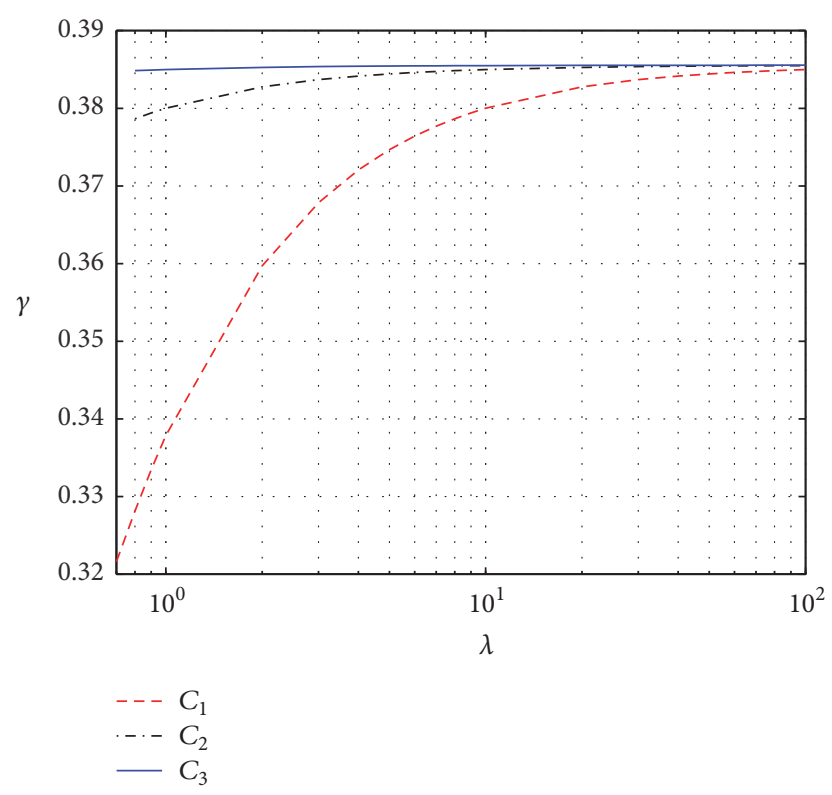

FIGURE 1: Behavior of $\gamma$ with the variation of $\lambda$ for a strictly proper filter obtained with Theorem 4 for different levels of sensor saturation.

In this example, the matrix $S_{1}$ has been considered as $S_{1}=$ $\operatorname{diag}(\lambda, \lambda), \lambda>0$, and three different situations for the saturation level $y_{0}$ have been considered:

$$
\begin{aligned}
& C_{1} \longrightarrow y_{0}^{\prime}=\left[\begin{array}{ll}
10^{-1} & 10^{-1}
\end{array}\right], \\
& C_{2} \longrightarrow y_{0}^{\prime}=\left[\begin{array}{ll}
10^{-2} & 10^{-2}
\end{array}\right], \\
& C_{3} \longrightarrow y_{0}^{\prime}=\left[\begin{array}{ll}
10^{-3} & 10^{-3}
\end{array}\right] .
\end{aligned}
$$

Figure 1 presents the behavior of $\gamma$ with the variation of $\lambda$ for a strictly proper filter (i.e., considering $D_{f}=0$ ) obtained with Theorem 4, considering three different levels of sensor saturation in the measured output $y$. For this system, the method in [17] provides a proper filter with $\gamma=0.4693$ for $y_{0}^{\prime}=\left[\begin{array}{ll}1 & 1\end{array}\right]$. It can be seen that Theorem 4 can provide smaller bounds to the $\mathscr{H}_{\infty}$ performance than [17] even for more restrictive situations in the output $y(t)$. Moreover, for each fixed value of $\lambda$, the curve for $C_{1}$ yields the small values for $\gamma$. This is expected since the case $C_{1}$ is the less restrictive situation.

In order to perform a time-domain simulation, let us consider the following input noise:

$$
w(t)=\sin (0.5 t) \exp (-0.1 t)\left[\begin{array}{l}
1 \\
1
\end{array}\right]
$$

Figure 2 shows the error signal obtained with a time simulation of the augmented system (10) considering two different levels of sensor saturation. The dashed red line depicts the error for a filter obtained with Theorem 4 with $C_{1}$ and $\lambda=0.7$, while the solid blue line shows the error-time response for a filter designed by Theorem 4 with $C_{3}$ and $\lambda=0.8$. It can be noted that the sensor saturation in the output $y$ can affect

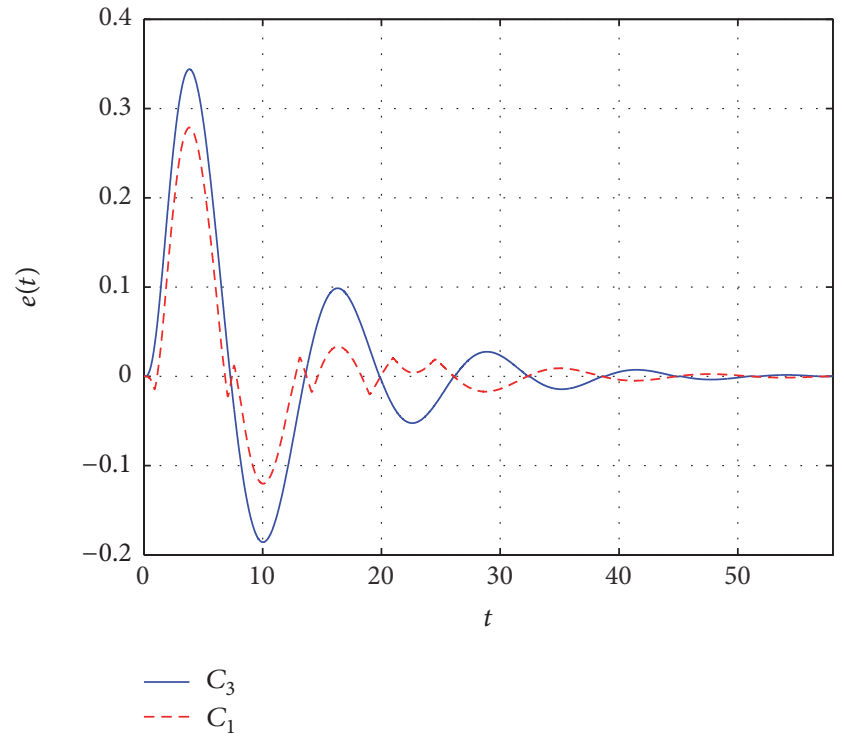

FIGURE 2: Error-time response for the augmented system (10) obtained for different levels of sensor saturation with initial condition $\widetilde{x}(0)=0$.
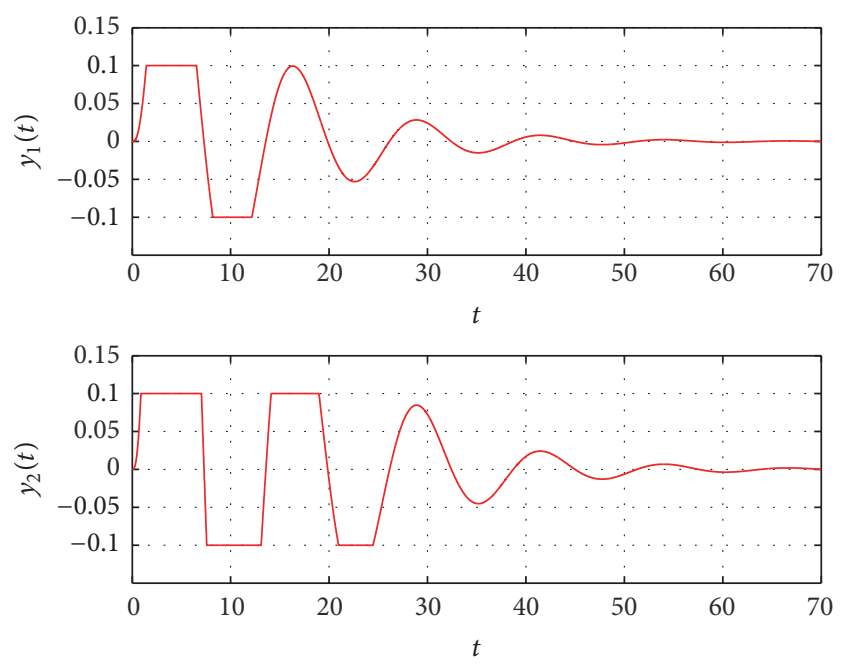

Figure 3: Behavior of the output $y$ with a saturation level $y_{0}^{\prime}=$ $\left[\begin{array}{ll}10^{-1} & 10^{-1}\end{array}\right]$.

directly the performance of the augmented system. Moreover, the filter obtained for $C_{1}$ provides a more attenuated error signal.

Figure 3 shows the behavior of the saturated output $y(t)$ considering case $C_{1}$ with $\lambda=0.7$ in Theorem 4 . Note that the component $y_{2}(t)$ is the most affected by the saturation level.

Example 8. Consider the following example adapted from [22] with matrices:

$$
\begin{aligned}
& A_{1}=\left[\begin{array}{cc}
-9.1 & 50 \\
-1 & -10
\end{array}\right], \\
& A_{2}=\left[\begin{array}{cc}
-0.1 & 50 \\
-1 & -10
\end{array}\right],
\end{aligned}
$$




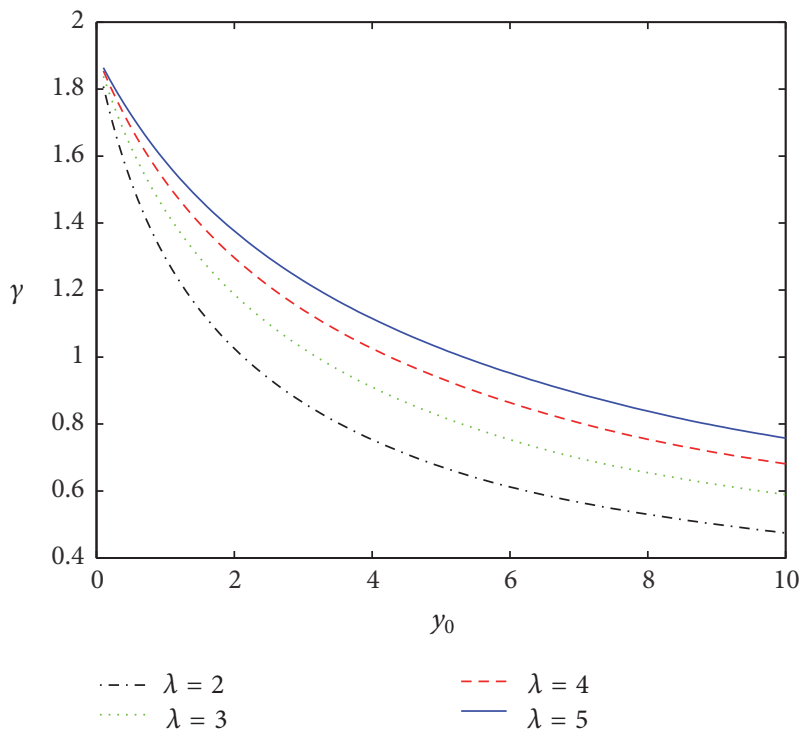

Figure 4: Behavior of $\gamma$ with the saturation level $y_{0}$ for different values of $\lambda$.

$$
\begin{aligned}
A_{3} & =\left[\begin{array}{cc}
-0.1 & 50 \\
-1 & -16
\end{array}\right], \\
A_{4} & =\left[\begin{array}{cc}
-0.1 & 50 \\
-1 & -4
\end{array}\right], \\
B_{i} & =\left[\begin{array}{ll}
0 & 1
\end{array}\right]^{\prime}, \\
C_{y i} & =\left[\begin{array}{ll}
1 & 0
\end{array}\right], \\
C_{z i} & =\left[\begin{array}{ll}
1 & 0
\end{array}\right], \\
D_{z i} & =0 .
\end{aligned}
$$

Figure 4 depicts the behavior of the bounds $\gamma$, for different values of $\lambda$ and considering different saturation levels $y_{0}$. One can see that the saturation level has an important role in the $\mathscr{H}_{\infty}$ performance. Moreover, the parameter $\lambda$ can be used as an extra degree of freedom to search for better solutions. As can be seen, $\lambda=2$ provided the lower bounds to the $\mathscr{H}_{\infty}$ performance in this case.

\section{Conclusion}

This paper has proposed new sufficient conditions for the design of full order robust linear filters for systems that are subject to sensor saturation. The designed filters guarantee an $\mathscr{H}_{\infty}$ performance for the augmented system. The proposed conditions were based on the use of a quadratic Lyapunov matrix and a generalized sector condition. Two different types of systems have been taken into consideration: precisely known systems and systems with time-varying uncertainties. It has been shown by numerical experiments that the saturation in the output $y(t)$ does affect the performance of the $\mathscr{H}_{\infty}$ robust filter. Moreover, the proposed technique proved to be efficient in taking into account the sensor saturation in the filter design problem. As future research, the author is investigating how to consider LPV filters to treat the systems with time-varying uncertainties.

\section{Conflicts of Interest}

The author declares that there are no conflicts of interest regarding the publication of this paper.

\section{References}

[1] H. K. Khalil, Nonlinear Systems, Prentice Hall, Upper Saddle River, NJ, USA, 3rd edition, 2002.

[2] M. J. Lacerda, S. Tarbouriech, G. Garcia, and P. L. D. Peres, "Ho filter design for nonlinear polynomial systems," Systems and Control Letters, vol. 70, pp. 77-84, 2014.

[3] V. J. Leite and M. F. Miranda, "Robust stabilization of discretetime systems with time-varying delay: an LMI approach," Mathematical Problems in Engineering, vol. 2008, Article ID 875609, 15 pages, 2008.

[4] T. Hu and Z. Lin, Control Systems with Actuator Saturation: analysis and design, Birkhäuser, Boston, Mass, USA, 2001.

[5] S. A. M. Martins and L. A. Aguirre, "Sufficient conditions for rate-independent hysteresis in autoregressive identified models," Mechanical Systems and Signal Processing, vol. 75, pp. 607-617, 2016.

[6] S. Tarbouriech, C. Prieur, and J. Gomes da Silva, "Stability analysis and stabilization of systems presenting nested saturations," Institute of Electrical and Electronics Engineers. Transactions on Automatic Control, vol. 51, no. 8, pp. 1364-1371, 2006.

[7] V. Kapila and K. M. Grigoriadis, Eds., Actuator Saturation Control, Control Engineering Series, Marcel Dekker, New York, NY, 2002.

[8] S. Tarbouriech, G. Garcia, and A. H. Glattfelder, Eds., Advanced Strategies in Control Systems with Input and Output Constraints, vol. 346 of Lecture Notes in Control and Information Sciences, Springer-Verlag, Berlin, Germany, 2007.

[9] B. D. O. Anderson and J. B. Moore, "Optimal Filtering," Prentice-Hall, Englewood, NJ, USA, 1979.

[10] J. C. Geromel, "Optimal linear filtering under parameter uncertainty," IEEE Transactions on Signal Processing, vol. 47, no. 1, pp. $168-175,1999$.

[11] D. F. Coutinho, C. E. de Souza, K. A. Barbosa, and A. Trofino, "Robust linear Ho filter design for a class of uncertain nonlinear systems: an LMI approach," SIAM Journal on Control and Optimization, vol. 48, no. 3, pp. 1452-1472, 2009.

[12] M. J. Lacerda, R. C. L. F. Oliveira, and P. L. D. Peres, “Robust $\mathrm{H} 2$ and $\mathrm{H} \infty$ filter design for uncertain linear systems via LMIs and polynomial matrices," Signal Processing, vol. 91, no. 5, pp. 1115-1122, 2011.

[13] C. E. de Souza, R. M. Palhares, and P. L. D. Peres, "Robust H8 filter design for uncertain linear systems with multiple timevarying state delays," IEEE Transactions on Signal Processing, vol. 49, no. 3, pp. 569-576, 2001.

[14] M. J. Lacerda, V. J. S. Leite, R. C. L. F. Oliveira, and P. L. D. Peres, "Delay-dependent robust H8 filter design for statedelayed discrete-time linear systems via homogeneous polynomial matrices," IET Control Theory Applications, vol. 7, no. 1, pp. 125-135, 2013. 
[15] P. Li, J. Lam, and G. Chesi, "On the synthesis of linear Ho filters for polynomial systems," Systems Control Letters, vol. 61, no. 1, pp. 31-36, 2012.

[16] C. El-Kasri, A. Hmamed, and F. Tadeo, "Reduced-order Ho filters for uncertain 2-D continuous systems, via LMIs and polynomial matrices," Circuits Systems and Signal Processing, vol. 33, pp. 1189-1214, 2014.

[17] B. Zhou, W. X. Zheng, Y.-M. Fu, and G.-R. Duan, "Ho filtering for linear continuous-time systems subject to sensor nonlinearities," IET Control Theory Applications, vol. 5, no. 16, pp. 1925-1937, 2011.

[18] S. Boyd, L. El Ghaoui, E. Feron, and V. Balakrishnan, Linear Matrix Inequalities in System and Control Theory, IAM Studies in Applied Mathematics, Philadelphia, Pa, USA, 1994.

[19] G. Garcia, S. Tarbouriech, J. M. G. da Silva Jr., and D. Eckhard, "Finite L2 gain and internal stabilisation of linear systems subject to actuator and sensor saturations," IET Control Theory Applications, vol. 3, pp. 799-812, 2009.

[20] G. Valmórbida, S. Tarbouriech, and G. Garcia, "State feedback design for input-saturating quadratic systems," Automatica, vol. 46, no. 7, pp. 1196-1202, 2010.

[21] P. Gahinet, "Explicit controller formulas for LMI-based Ho synthesis," Automatica, vol. 32, no. 7, pp. 1007-1014, 1996.

[22] Z. Li, J. Li, J. Yu, and X. Zhao, "Robust filter design for a class of uncertain systems with D stability constraints under a unified framework," Journal of the Franklin Institute, vol. 353, no. 16, pp. 4233-4252, 2016.

[23] J. Löfberg, "YALMIP: A toolbox for modeling and optimization in MATLAB," in in Proceedings of the 2004 IEEE International Symposium on Computer Aided Control Systems Design, pp. 284-289, Taipei, Taiwan, September 2004.

[24] J. F. Sturm, "Using SeDuMi 1.02, a MATLAB toolbox for optimization over symmetric cones," Optimization Methods and Software, vol. 11, no. 1-4, pp. 625-653, 1999, http://sedumi.ie .lehigh.edu/.

[25] Y.-Y. Cao, Z. Lin, and B. M. Chen, "An output feedback H8 controller design for linear systems subject to sensor nonlinearities," in IEEE Transactions on Circuits and Systems Part I: Fundamental Theory and Applications, vol. 50, pp. 914-921, 2003. 


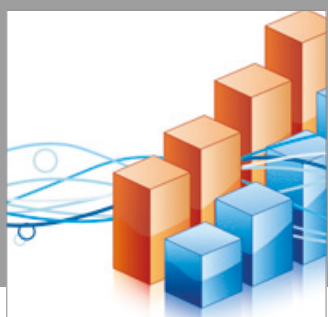

Advances in

Operations Research

vatersals

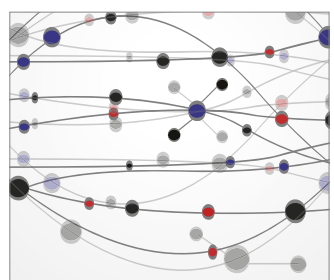

\section{The Scientific} World Journal
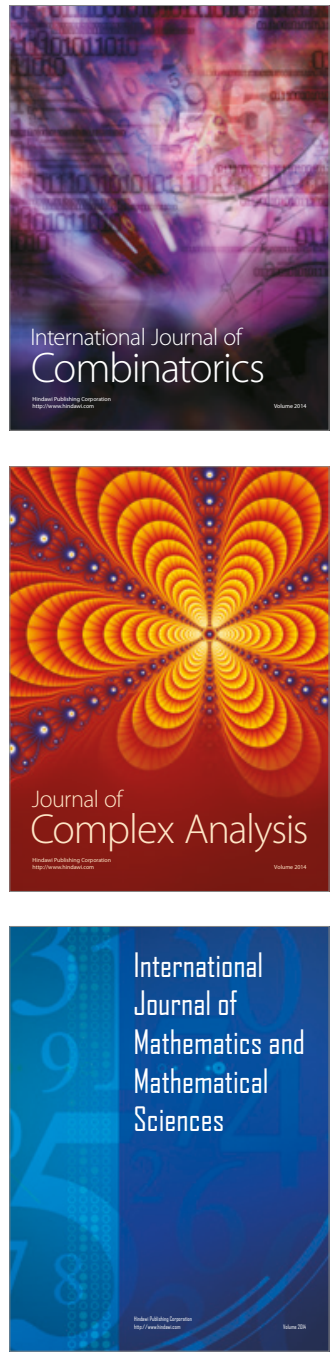
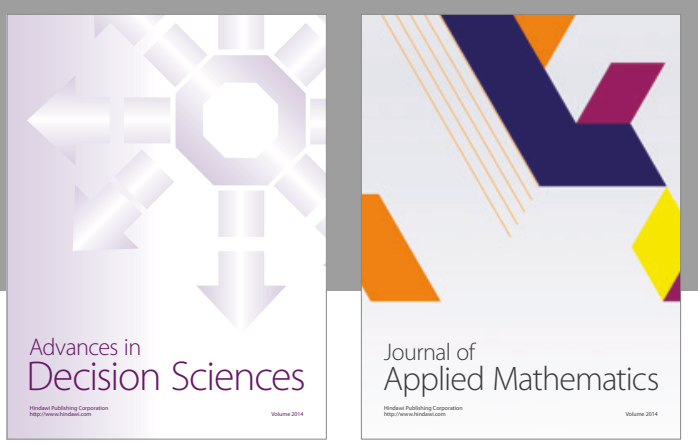

Algebra

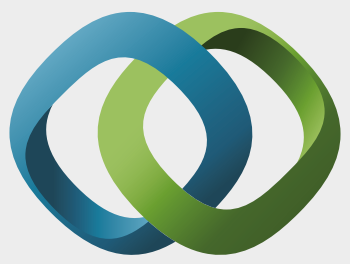

\section{Hindawi}

Submit your manuscripts at

https://www.hindawi.com
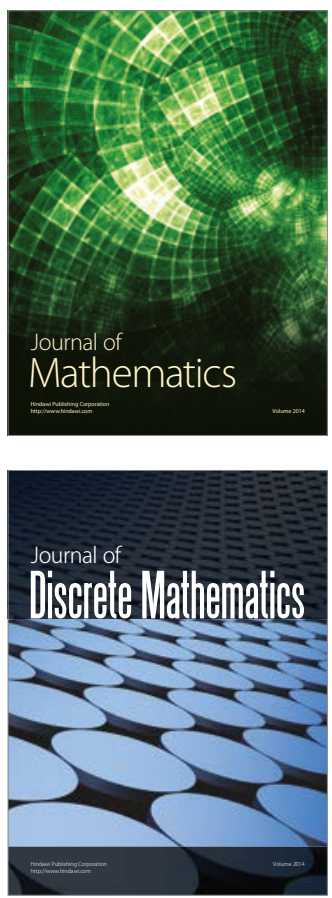

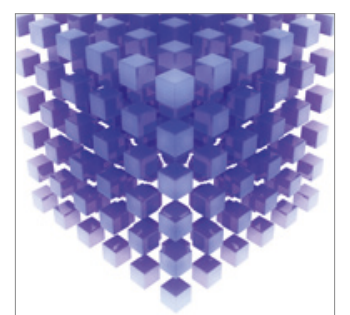

Mathematical Problems in Engineering
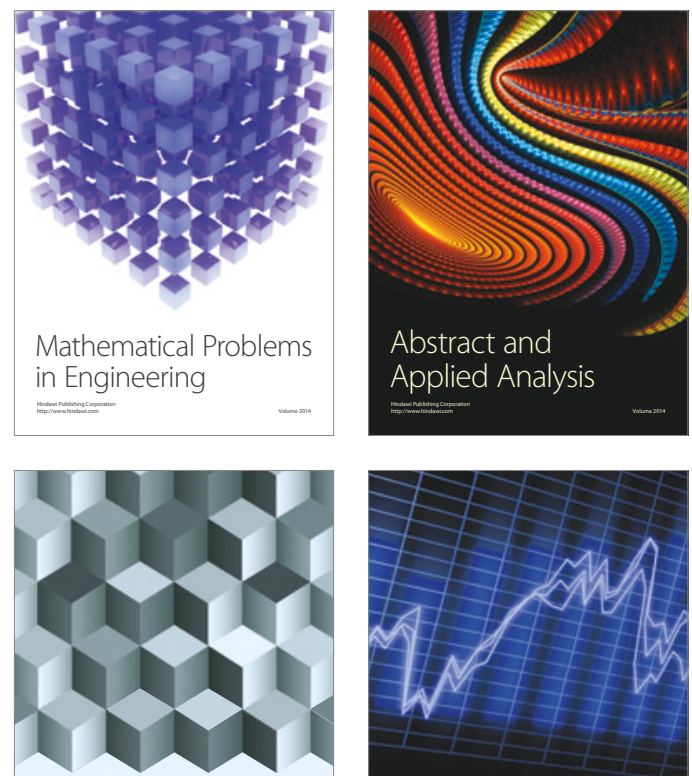

Journal of

Function Spaces

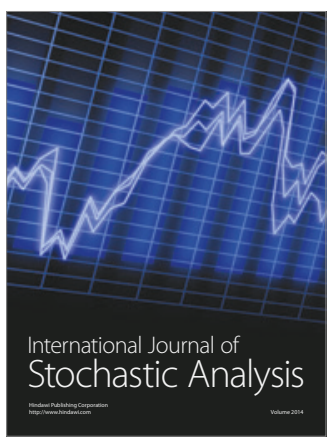

Probability and Statistics
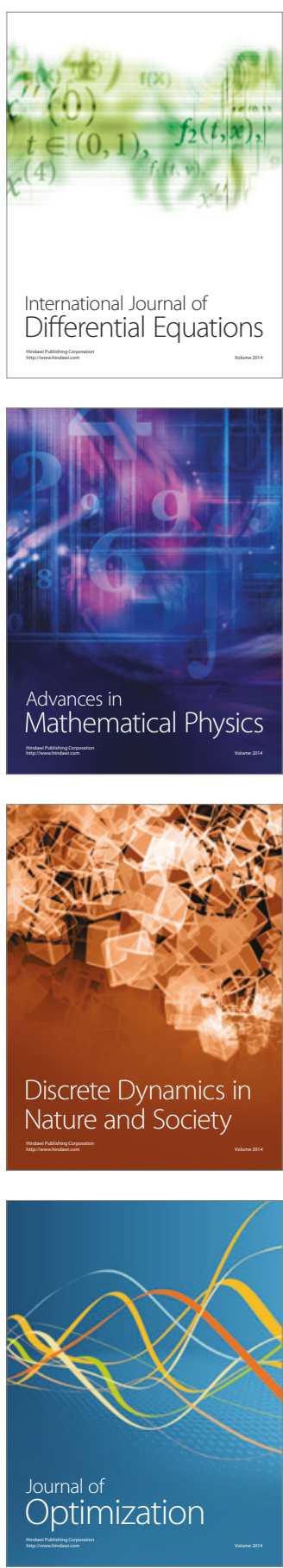